\title{
Identification of APN/CD13 as the target antigen of FU3, a human monoclonal antibody that recognizes malignant fibrous histiocytoma
}

\author{
MIKIKO AOKI, KAZUKI NABESHIMA, HIROYUKI HAYASHI, \\ MAKOTO HAMASAKI and HIROSHI IWASAKI \\ Department of Pathology, Fukuoka University School of Medicine and Hospital, Jonan-ku, \\ Fukuoka 814-0180, Japan
}

Received February 27, 2013; Accepted April 22, 2013

DOI: 10.3892/ijo.2013.1940

\begin{abstract}
Malignant fibrous histiocytoma (MFH), a highgrade, undifferentiated sarcoma, is highly aggressive, resistant to radiochemotherapy and associated with poor prognosis. There are no specific immunohistochemical markers for its diagnosis. The MFH cell line SFT7913 served as and immunogen for the generation of the FU3 monoclonal antibody in our laboratory. FU3 reacted strongly with MFH cells and with perivascular mesenchymal cells. In this study, we demonstrated that the antigen recognized by FU3 was identical to aminopeptidase N (APN/CD13) using FU3 immunoaffinity chromatography and $\mathrm{N}$-terminal amino acid sequencing. Frequent $(80 \%)$ and high-grade $(>50 \%$ of cells) expression of APN/CD13 was observed in MFH, although low-grade expression was seen in some other sarcomas. Moreover, small interfering RNA (siRNA) that specifically targets APN/CD13 significantly suppressed MFH cell invasion in vitro. The newly developed monoclonal antibody FU3 specifically recognizes CD13 on MFH cells. Decreased expression of CD13, mediated by siRNA-mediated knockdown, attenuated the invasive capacity of MFH cells. Thus, results indicate that APN/CD13 could be an important diagnostic biomarker and therapeutic target for MFH.
\end{abstract}

\section{Introduction}

Malignant fibrous histiocytoma (MFH), also called high grade undifferentiated sarcoma, collectively represent the most common types of sarcoma in the fifth and sixth decades of life. The overall incidence among adults approximates to 1-2 cases per 100,000 patients. Most MFH occur in the extremities and

Correspondence to: Professor K. Nabeshima, Department of Pathology, Fukuoka University School of Medicine and Hospital, 7-45-1 Nanakuma, Jonan-ku, Fukuoka 814-0180, Japan

E-mail:kaznabes@fukuoka-u.ac.jp

Key words: aminopeptidase N, APN/CD13, human monoclonal antibody FU3, malignant fibrous histiocytoma deep soft tissue (1). Approximately $5 \%$ of patients have metastases at presentation and MFH is aggressive with an overall 5 -year survival probability of 50-60\% (1). Surgical removal is presently the sole effective treatment and the goal of surgery is complete resection with negative margin.

Histopathologically, MFH shows a wide variety of morphological patterns. MFH commonly presents with marked cytological and nuclear pleomorphism, often with bizarre tumor giant cells, admixed with spindle cells and frequently with rounded histiocyte-like cells in varying proportions (2). MFH shows no evidence of true monocyte/macrophage/histiocytic differentiation. Several hypotheses suggest MFH arises from fibroblasts or primitive mesenchymal cells but current research does not show a definable line of differentiation. The diagnosis is controversial, but various cases are eligible for consideration as MFH (high grade undifferentiated sarcoma). At present, there are no useful immunohistochemical markers for the diagnosis of MFH; therefore, it is difficult to search for the origin of $\mathrm{MFH}$.

Our laboratory generated monoclonal antibody FU3 using an MFH cell line as an immunogen. FU3 reacted strongly with the surface membrane of cultured MFH cells and with perivascular mesenchymal cells in frozen tissue sections. Accordingly, MFH may share common antigenicity with perivascular mesenchymal cells (3). Immuno-electron-microscopic studies demonstrated FU3-positive reactivity on the surface of cell membranes, which suggests that FU3 recognizes cell surface antigens (4-6).

The aim of this study was to identify the antigen recognized by FU3 antibody. Furthermore, we examined whether the antigen could be effectively applied to diagnosis and treatment of MFH.

\section{Materials and methods}

Cell culture. The MFH cell line SFT8503 was established in our laboratory, as described previously (6). The cell line was maintained in growth medium, Dulbecco's modified Eagle's medium/ Ham's F-12 (Wako, Japan), supplemented with $10 \%$ fetal calf serum, streptomycin $(50 \mu \mathrm{g} / \mathrm{ml})$ and penicillin $\mathrm{G}(50 \mathrm{U} / \mathrm{ml})$. 
Protein extraction and western blotting. The cultured cells were lysed in RIPA lysis buffer $(50 \mathrm{mM}$ Tris-HCl, $\mathrm{pH} 7.4$, $150 \mathrm{mM} \mathrm{NaCl}, 1 \mathrm{mM}$ EDTA, $1 \%$ NP-40; Millipore, Bedford, $\mathrm{MA}$ ) and the lysed cells were sonicated on ice for $5 \mathrm{~min}$ three times and centrifuged at $15,000 \mathrm{rpm}$ for $20 \mathrm{~min}$ at $4^{\circ} \mathrm{C}$. The resultant supernatants were subjected to sodium dodecyl sulfate-polyacrylamide gel electrophoresis (SDS-PAGE). After electrophoresis, the proteins were transferred electrophoretically to Immobilon membrane (Millipore). Non-specific sites were blocked with $5 \%$ dry fat milk in Tris-buffered saline (TBS) at $37^{\circ} \mathrm{C}$ for $1 \mathrm{~h}$ and the membrane was incubated overnight at $4^{\circ} \mathrm{C}$ with monoclonal FU3 antibody or with commercially available CD13 antibody (Clone 38C12, ThermoScientific, Cheshire, UK). After washing with TBS-T (TBS containing $0.05 \%$ Tween-20), the membrane was incubated for $1 \mathrm{~h}$ with peroxidase-conjugated anti-mouse IgG. Color was developed with chemiluminescence reagents according to the instructions supplied by the manufacturer (DuPont New England Nuclear, Boston, MA).

Immunoaffinity chromatography. Monoclonal antibody FU3, which was generated against MFH cells using a mouse hybridoma technique as previously described $(3,4)$, was used to prepare an affinity column (7). We used cyanogen bromide CNBr-activated Sepharose 4B (GE Healthcare Ltd., UK) as an immunoaffinity matrix coupling to mouse FU3 antibody. The column was washed with phosphate buffered saline (PBS). Antigens bound to the matrix were then eluted from the column with $0.2 \mathrm{M}$ glycine ( $\mathrm{pH} 2.3$ ). The eluted material was immediately neutralized with PBS and stored at $4^{\circ} \mathrm{C}$. The eluted protein fraction of the column was purified by SDS-PAGE and immunoblotting. The purified protein was subjected to $\mathrm{N}$-terminal amino acid sequencing (Takara Bio Inc. Otsu, Japan).

Tissue samples. Our study included formalin-fixed, paraffinembedded sections from $25 \mathrm{MFH}$ [10 men, 15 women; age range, 26-81 (mean, 71) years], 9 synovial sarcoma [2 men, 7 women; age range, 20-88 (mean, 43) years], 10 liposarcoma [ 4 men, 6 women; age range, 20-74 (mean, 55) years], 10 leiomyosarcoma (4 men, 6 women; age range, 25-83 (mean, 62) years], 11 chondrosarcoma [7 men, 4 women; age range, 12-63 (mean, 42) years] and 5 osteosarcoma [5 women; age range, 14-57 (mean, 56) years], diagnosed at the Department of Pathology, Fukuoka University, Japan.

Immunohistochemistry. Immunohistochemical staining was performed using 4- $\mu \mathrm{m}$ thick paraffin-embedded sections, were deparaffinized and heated in a microwave oven $(700 \mathrm{~W})$ for $10 \mathrm{~min}$ to expose antigens in $10 \mathrm{mM}$ Na-citrate buffer $(\mathrm{pH} 6.0$; for CD13) or $1 \mathrm{mM}$ EDTA/10 mM Tris-HCl buffer (pH 9.0; for FU3). The exposed antigen was detected using the labeled streptavidin-biotin method. The reaction was identified with naphthol AS-BI phosphate and counterstained with Mayer's hematoxylin.

The staining results were evaluated semiquantitatively by two independent observers. Immunostaining was considered negative if stained tumor cells were $<10 \%$. In specimens considered positive, staining of the tumor was quantitated on a scale from 1-4 based on the percentage of positive tumor cells. The

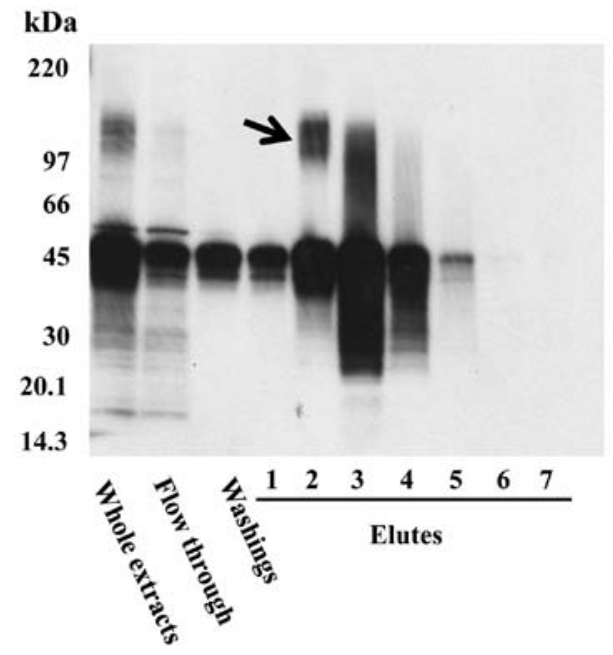

Figure 1. Western immunoblot analysis of whole ectracts (lane 1), flow through (lane 2), washings (lane 3) and elutes (lanes 4-10) immunostained with monoclonal antibody FU3 and showing the 150-kDa band.

scale was structured as follows: $1+, 10-25 \%$ of cells positive; $2+$, $25-50 \%$ of cells positive; $3+, 50-75 \%$ of cells positive; $4+,>75 \%$ of cells positive. In specimens considered high expression cases, staining of the tumor was quantified on scales $3+$ and $4+$.

Small interfering RNA (siRNA). SFT8503 cells were grown to sub-confluence and treated with small interfering RNA (siRNA) for CD13 (Smart Pool, Dharmacon, Chicago, IL) or control siRNA (B-Bridge International, Sunnyvale, CA) using Lipofeamine 2000 (Invitrogen, Carlsbad, CA) accroding to the manufacturer's instructions.

In vitro invasion assay. In vitro Matrigel invasion assay was performed by using 24-well Chemotaxicell chambers (pore size, $8 \mu \mathrm{m}$, Kubota Co., Tokyo, Japan) on 24-well culture plate. The upper and lower side of each chamber was coated with Matrigel (25 $\mu \mathrm{g} /$ filter, BD Biosciences). Hepatocyte growth factor (HGF; Peprotech Inc. Rocky Hill, NJ) was used as a chemoattractant. After incubation of cells for $72 \mathrm{~h}$, the filters were fixed with formalin and stained with hematoxylin and the total number of cells that had invaded the Matrigel-coated filter were counted.

\section{Results}

Immunoaffinity chromatography using FU3 antibody. Extracted proteins from SFT8503 MFH cells were purified using immunoaffinity chromatography with FU3 antibody, followed by immunoblotting. The N-terminal amino acid sequencing of the $150-\mathrm{kDa}$ band (Fig. 1, arrow) revealed A-K-G-F-Y-I-S-K-S-L, which is identical to that of aminopeptidase N (APN)/CD13, known as an important zinc-dependent metallo-exopeptidase.

The FU3 recognizing antigen is possibly aminopeptidase $N$ (CD13). To confirm the identity of FU3-reactive antigen as APN/CD13 we used immunoblotting and siRNA methods. First, by immunoblotting SFT8503 MFH whole cell extracts and an eluted protein from immunoaffinity chromatography 
a

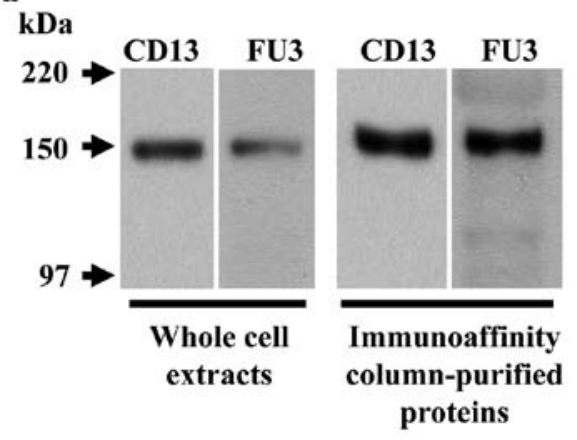

b

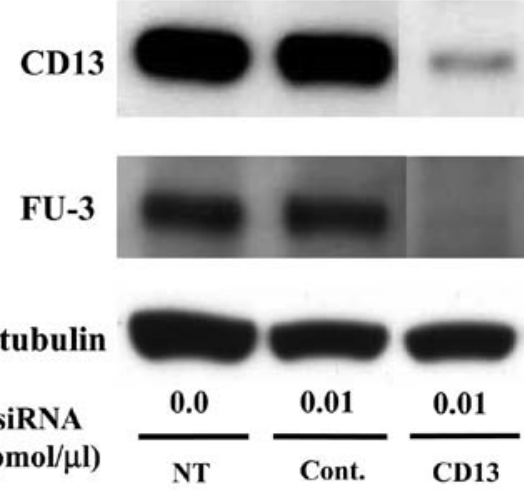

Figure 2. FU3 recognizing antigen corresponds to the aminopeptidase N (CD13). (a) Western immunoblot analysis of whole cell extracts and immunoaffinity column-purified proteins. Both CD13 and FU3 recognizes identical 150-kDa protein. (b) CD13 siRNA (0.01 pmol/ $\mu 1)$ treatment causes downregulation of both CD13 and FU-3-reactive protein at $72 \mathrm{~h}$. NT, non-treated; cont, control siRNA.

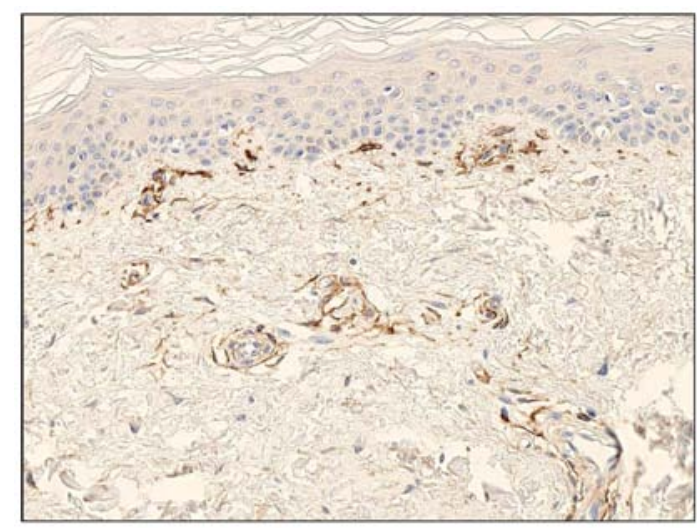

Figure 3. Reactivity of normal skin cells with CD13. Perivascular mesenchymal cells in the normal dermis show staining, whereas epidermal cells and endothelial cells remain unstained. CD13-positive fibroblasts are also noted in part. columns, both CD13 and FU3 antibodies recognized an identical 150-kDa band (Fig. 2a).

Second, CD13-specific siRNA treatment $(0.01 \mathrm{pmol} / \mu \mathrm{l})$ of SFT8503 MFH cells caused significant downregulation of both CD13 and FU3-reactive $150 \mathrm{kDa}$ proteins (Fig. 2b.). In view of the evidence, we considered that the FU3 reactive antigen was identical to APN/CD13.

CD13 expression in normal skin. Immunohistochemically, CD13 antibody stained perivascular mesenchymal cells, represented by small spindle or polygonal cells around small blood vessels, in normal skin (Fig. 3). A small number of fibroblasts also reacted with CD13 antibody; however, the stratified squamous epithelium and endothelial cells were not CD13 antibody-reactive. These findings were similar to those of FU3 reactive cells (3-6), supporting of the identification of FU3 antigen as APN/CD13.
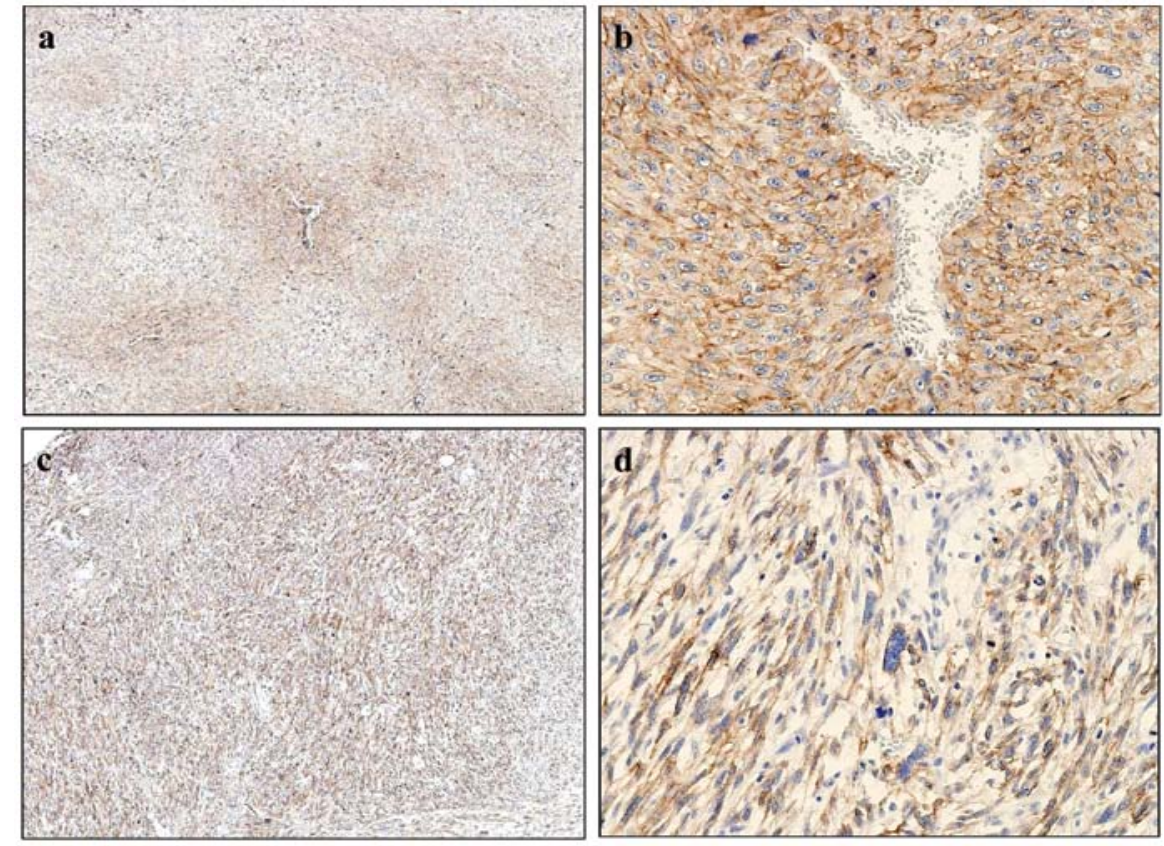

Figure 4. Immunohistochemical expression of CD13 in MFH. Representative cases of perivascular (a and b) and diffuse (c and d) patterns. The perivascular type shows strong cytoplasmic staining along the membrane (b). The diffuse type shows moderate cytoplasmic reactivity (d). Original magnifications (a and c), $\mathrm{x} 40 ;(\mathrm{b}$ and $\mathrm{d}), \mathrm{x} 200$. 
a

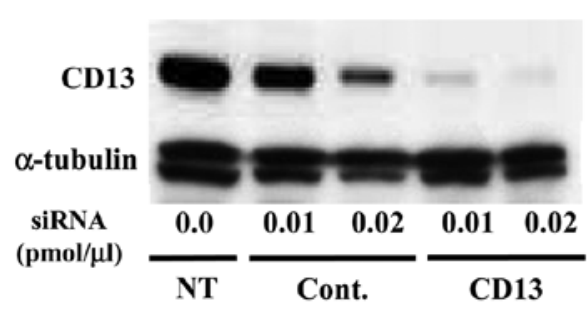

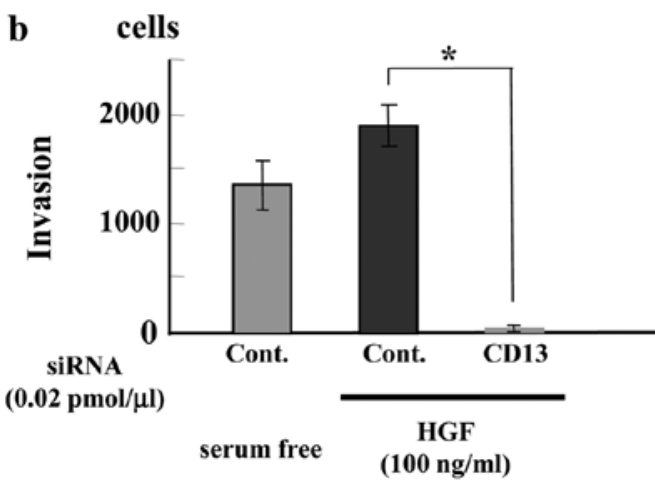

Figure 5. Expression of CD13 in SFT8503 cells and effect of CD13 siRNA on tumor invasion. (a) CD13 protein expression level by western blotting. Treatment with CD13 siRNA downregulated the level more severely at $0.02 \mathrm{pmol} / \mu 1$ than $0.01 \mathrm{pmol} / \mu 1$ at $72 \mathrm{~h}$. (b) Tumor cell invasion of Matrigel was significantly suppressed by transfection of CD13 siRNA $(0.02 \mathrm{pmol} / \mu \mathrm{l})$ at $72 \mathrm{~h}$. NT, non-treated; cont, control si RNA. ${ }^{*} \mathrm{P}<0.01$ by Student's t-test.

Table I. CD13 expression in soft tissue tumors.

\begin{tabular}{lrrc}
\hline & $\begin{array}{c}\text { No. of } \\
\text { cases }\end{array}$ & $\begin{array}{c}\text { Positive } \\
\text { cases }(\%)\end{array}$ & $\begin{array}{c}\text { High expression } \\
\text { cases }(>50 \% \text { of cells })\end{array}$ \\
\hline MFH & 25 & $20(80)$ & $17(68 \%)$ \\
Synovial sarcoma & 9 & $5(56)$ & $0(0 \%)$ \\
Liposarcoma & 10 & $0(0)$ & $0(0 \%)$ \\
Leiomyosarcoma & 10 & $3(30)$ & $2(20 \%)$ \\
Chondrosarcoma & 11 & $0(0)$ & $0(0 \%)$ \\
Osteosarcoma & 5 & $5(100)$ & $0(0 \%)$ \\
\hline
\end{tabular}

Positive cases, $1+$ to $4+$; high expression cases, $>3+$.

CD13 expression in MFH and soft tissue tumors. Expression of CD13 was examined immunohistochemically in $70 \mathrm{soft}$ tissue tumors wich included $25 \mathrm{MFH}$ and 45 other soft tissue tumors (Fig. 4 and Table I). Twenty cases of MFH (80\%) were positive for CD13 and of these, there were 17 (68\%) high expression cases. In MFH, CD13 expression pattern were classified into two types; perivascular (Fig. 4a and b) and diffuse types (Fig. $4 \mathrm{c}$ and d). Tissues of the perivascular type exhibited intense cytoplasmic and membrane staining of CD13 (Fig. 4b). In the diffuse type, almost all MFH cells had positively stained cytoplasms (Fig. 4d). Positive reactivity with CD13 was observed in several synovial sarcoma, leiomyosarcoma and osteosarcoma, but high-expression was found only in two cases of leiomyosarcoma (Table I).

Inhibition of MFH (SFT8503) cell invasion by CD13 siRNA. To investigate a biological role for APN/CD13 in MFH, we examined effect of CD13 siRNA treatment on MFH cell invasion. We used HGF as a chemoattractant factor because overexpression of HGF has been reported in MFH $(8,9)$. Treatment of SFT8503 MFH cells with CD13 siRNA (0.01 and $0.02 \mathrm{pmol} / \mu \mathrm{l}$ ) downregulated $\mathrm{CD} 13$ expression in a dosedependent manner at $48 \mathrm{~h}$ (data not shown) and at $72 \mathrm{~h}$ (Fig. 5a) after transfection. HGF (100 ng/ml) induced greater SFT8503 cell invasion than serum-free medium. In the presence of CD13-targeted siRNA, HGF-stimulated MFH cell invasion was significantly attenuated as compared with that observed with control siRNA (Fig. 5b).

\section{Discussion}

Development of an MFH recognizing FU3 antibody provided some important findings, especially on the cellular origin of MFH (4-6). The antibody, however, has not been widely used, one of the reasons may be that FU3 antibody is available only on frozen tissue sections but not on paraffin-embedded specimens. In this study, we demonstrated that the FU3 antibody recognizies an antigen identical to APN/CD13 using immunoaffinity chromatography and direct $\mathrm{N}$-terminal amino acid sequencing. Immunohistochemically, greater amounts of APN/CD13 were observed more frequently in MFH tissues as compared with that observed in other sarcomas in paraffinembedded specimens. Moreover, MFH cell invasion was significantly suppressed by transfection of APN/CD13 siRNA. The results from this study may point toward the use of APN/ $\mathrm{CD} 13$, the FU3 antigen, as an important biomarker in the diagnosis and treatment of patients with MFH.

APN/CD13, a 150-kDa metalloproteinase, is a multifunctional cell surface aminopeptidase. The human APN gene has been mapped to chromosome 15q25-26. APN/CD13 expression has been reported in hematopoietic cells of myeloid origin, fibroblasts, synaptic membranes in the central nervous system and epithelial cells of liver, kidney and intestine $(10,11)$. High expression levels of APN/CD13 has been detected in various epithelial tumors and its expression correlates with increased clinical malignant behavior in pancreatic carcinoma and in colon and non-small lung cancer (12-14).

There is little information on the expression of APN/CD13 and its role in MFH. An immunohistochemical study of MFH demonstrated a positive reaction to APN/CD13 in six of ten cases (15). Another report showed four cell lines derived from an MFH expressed APN/CD13, using flow cytometric analysis (16). Immunohistochemically, APN/CD13 antibody showed strong reactivity with perivascular mesenchymal cells in the normal skin tissue and with MFH cells, to a similar extent seen with FU3. These findings lend support to our result that the FU3 recognizing antigen is identical to APN/CD13. Although we can not directly compare our results with those 
of previous results of APN/CD13 expression (15), owing to the differences in assessment methods, our findings were roughly consistent with previous studies on other soft tissues sarcomas. All our five cases of osteosarcoma were positive for APN/CD13, but no high expression cases were observed. Only two cases of leiomyosarcoma showed high expression. APN/CD13 immunostaining may be applicable to narrow the differential diagnosis of soft tissue sarcomas to MFH.

Our immunohistochemical study in normal skin demonstrated APN/CD13 expression in the perivascular cells and in some dermal fibroblasts. APN/CD13 expression in perivascular cells has also been reported in lung tissue; the majority of these CD13-positive cells were slender perivascular fibroblastic cells (17). Dermal fibroblasts express APN/CD13 to a relatively great degree in vitro $(18,19)$. On the basis of immunoreactivity, MFH cells may have intimate relationship with perivascular cells and fibroblasts.

APN/CD13 might participate in tumor progression by regulating processes such as tumor invasion and angiogenesis (12,20-24). Our study, however, is the first to show that downregulation of APN/CD13 expression leads to marked suppression of invasion by MFH cells, although this is based on only in vitro data. In a previous study with osteosarcoma cell lines, CD13 siRNA treatment caused reduced cellular attachment to and increased proteolytic degeneration of the extracellular matrix (25). Anti-APN/CD13 antibody reduced the migratory activity of human dermal fibroblasts (19). These reduced activities may also occur in MFH cells and these possibilities are now under investigation in our laboratory.

Since the discovery in 1976 of the first APN enzymatic inhibitor bestatin, many APN inhibitors have been developed (26). Bestatin is already used clinically for the treatment of adult acute non-lymphocytic leukemia via peroral administration. Bestatin-mediated suppression of APN/CD13 activity in an APN/CD13-expressing ovarian carcinoma cells led to reduced migration, proliferation and peritoneal dissemination of tumor cells in a mouse model, which resulted in prolonged survival (27). Bestatin may also represent a new approach for improving the therapeutic efficacy of radiotherapy for uterine cervical carcinoma (28) and for enhancing paclitaxel chemosensitivity in ovarian carcinoma (29). Recently, some novel potent APN/CD13 inhibitors have also been reported, several of which show better inhibitory activity than bestatin against APN on human carcinoma cells $(30,31)$.

In conclusion, our study indicates that APN/CD13 may be useful for diagnosing $\mathrm{MFH}$ and importantly, might serve as a new molecular target for therapy for patients with MFH.

\section{Acknowledgements}

We acknowledge the expert technical assistance of Ms. M. Onitsuka, M. Ishiguro and C. Fujita in immunohistochemical staining and in vitro studies.

\section{References}

1. Gustafson P: Soft tissue sarcoma. Epidemiology and prognosis in 508 patients. Acta Orthop Scand (Suppl) 259: 1-31, 1994.
2. Fletcher CD: Pleomorphic malignant fibrous histiocytoma: fact or fiction? A critical reappraisal based on 159 tumors diagnosed as pleomorphic sarcoma. Am J Surg Pathol 16: 213-228, 1992.

3. Isayama T, Iwasaki $\mathrm{H}$ and Kikuchi $\mathrm{M}$ : The origin of malignant fibrous histiocytoma immunohistochemical analysis with monoclonal antibodies. Medical Bulltein of Fukuoka University 14: 191-203, 1987.

4. Iwasaki H, Isayama T, Johzaki $\mathrm{H}$ and Kikuchi M: Malignant fibrous histiocytoma. Evidence of perivascular mesenchymal cell origin immunocytochemical studies with monoclonal anti-MFH antibodies. Am J Pathol 128: 528-537, 1987.

5. Iwasaki H, Yoshitake K, Ohjimi Y, et al: Malignant fibrous histiocytoma. Proliferative compartment and heterogeneity of 'histiocytic' cells. Am J Surg Pathol 16: 735-745, 1992.

6. Iwasaki H, Isayama T, Ohjimi Y, et al: Malignant fibrous histiocytoma. A tumor of facultative histiocy tes showing mesenchymal differentiation in cultured cell lines. Cancer 69: 437-447, 1992.

7. Ellis SM, Nabeshima K and Biswas C: Monoclonal antibody preparation and purification of a tumor cell collagenase-stimulatory factor. Cancer Res 49: 3385-3391, 1989.

8. Yamamoto T, Marui T, Akisue T, et al: Coexpression of hepatocyte growth factor and its receptor c-Met correlates with high MIB-1 proliferative index in malignant fibrous histiocytoma. Pathol Res Pract 200: 397-402, 2004.

9. Wallenius V, Hisaoka M, Helou K, et al: Overexpression of the hepatocyte growth factor (HGF) receptor (Met) and presence of a truncated and activated intracellular HGF receptor fragment in locally aggressive/malignant human musculoskeletal tumors. Am J Pathol 156: 821-829, 2000.

10. Luan $\mathrm{Y}$ and $\mathrm{Xu} \mathrm{W}$ : The structure and main functions of aminopeptidase N. Curr Med Chem 14: 639-647, 2007.

11. Zhang X and Xu W: Aminopeptidase N (APN/CD13) as a target for anti-cancer agent design. Curr Med Chem 15: 2850-2865, 2008.

12. Ishii K, Usui S, Sugimura Y, et al: Aminopeptidase N regulated by zinc in human prostate participates in tumor cell invasion. Int J Cancer 92: 49-54, 2001.

13. Ikeda N, Nakajima Y, Tokuhara T, et al: Clinical significance of aminopeptidase N/CD13 expression in human pancreatic carcinoma. Clin Cancer Res 9: 1503-1508, 2003.

14. Tokuhara T, Hattori N, Ishida H, et al: Clinical significance of aminopeptidase $\mathrm{N}$ in non-small cell lung cancer. Clin Cancer Res 12: 3971-3978, 2006.

15. Mechtersheimer G and Moller P: Expression of aminopeptidase $\mathrm{N}$ (CD13) in mesenchymal tumors. Am J Pathol 137: 1215-1222, 1990.

16. Mori A, Tagawa T, Kamei T, Murata T, Inui M and Ohse S: Characterization of four cell lines derived from a human malignant fibrous histiocytoma of the maxillary sinus. Oral Oncol 37: 527-536, 2001.

17. Ichimura E, Yamada M, Nishikawa $K$, Abe $F$ and Nakajima T: Immunohistochemical expression of aminopeptidase N (CD13) in human lung squamous cell carcinomas, with special reference to Bestatin adjuvant therapy. Pathol Int 56: 296-300, 2006.

18. Gabrilovac J, Cupic B, Breljak D, Zekusic M and Boranic M: Expression of CD13/aminopeptidase N and CD10/neutral endopeptidase on cultured human keratinocytes. Immunol Lett 91: 39-47, 2004.

19. Lai A, Ghaffari A and Ghahary A: Inhibitory effect of antiaminopeptidase N/CD13 antibodies on fibroblast migration. Mol Cell Biochem 343: 191-199, 2010.

20. Mishima Y, Terui Y, Sugimura N, et al: Continuous treatment of bestatin induces anti-angiogenic property in endothelial cells. Cancer Sci 98: 364-372, 2007.

21. Pasqualini R, Koivunen E, Kain R, et al: Aminopeptidase $\mathrm{N}$ is a receptor for tumor-homing peptides and a target for inhibiting angiogenesis. Cancer Res 60: 722-727, 2000.

22. Saiki I, Fujii H, Yoneda J, et al: Role of aminopeptidase N (CD13) in tumor-cell invasion and extracellular matrix degradation. Int J Cancer 54: 137-143, 1993.

23. Fujii H, Nakajima M, Saiki I, Yoneda J, Azuma I and Tsuruo T: Human melanoma invasion and metastasis enhancement by high expression of aminopeptidase N/CD13. Clin Exp Metastasis 13: 337-344, 1995.

24. Wulfanger J, Schneider H, Wild P, et al: Promoter methylation of aminopeptidase N/CD13 in malignant melanoma. Carcinogenesis 33: 781-790, 2012.

25. Kido A, Krueger S, Haeckel C and Roessner A: Inhibitory effect of antisense aminopeptidase N (APN/CD13) cDNA transfection on the invasive potential of osteosarcoma cells. Clin Exp Metastasis 20: 585-592, 2003. 
26. Umezawa H, Aoyagi T, Suda H, Hamada M and Takeuchi T: Bestatin, an inhibitor of aminopeptidase B, produced by actinomycetes. J Antibiot (Tokyo) 29: 97-99, 1976.

27. Terauchi M, Kajiyama H, Shibata K, et al: Inhibition of APN/ CD13 leads to suppressed progressive potential in ovarian carcinoma cells. BMC Cancer 7: 140, 2007.

28. Tsukamoto H, Shibata K, Kajiyama H, Terauchi M, Nawa A and Kikkawa F: Aminopeptidase N (APN)/CD13 inhibitor, Ubenimex, enhances radiation sensitivity in human cervical cancer. BMC Cancer 8: 74, 2008.

29. Yamashita M, Kajiyama $\mathrm{H}$, Terauchi $\mathrm{M}$, et al: Involvement of aminopeptidase $\mathrm{N}$ in enhanced chemosensitivity to paclitaxel in ovarian carcinoma in vitro and in vivo. Int $\mathbf{J}$ Cancer 120 2243-2250, 2007.
30. Zhang X, Zhang L, Zhang J, et al: Design, synthesis and preliminary activity evaluation of novel 3-amino-2-hydroxyl3-phenylpropanoic acid derivatives as aminopeptidase N/CD13 inhibitors. J Enzyme Inhib Med Chem28: 545-551, 2013.

31. Su L, Jia Y, Zhang L, Xu Y, Fang H and Xu W: Design, synthesis and biological evaluation of novel amino acid ureido derivatives as aminopeptidase N/CD13 inhibitors. Bioorg Med Chem 20: 3807-3815, 2012 